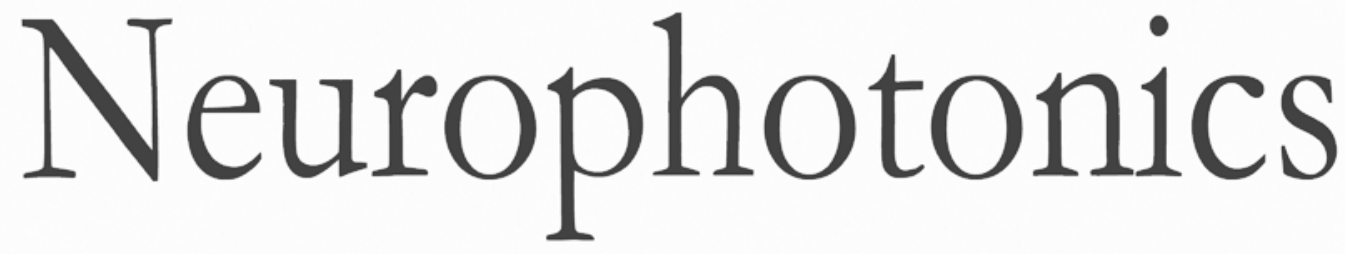

\title{
Graph theoretical approach to functional connectivity in prefrontal cortex via fNIRS
}

\author{
Zahra Einalou
}

Keivan Maghooli

Seyaed Kamaledin Setarehdan

Ata Akin 


\title{
Graph theoretical approach to functional connectivity in prefrontal cortex via fNIRS
}

\author{
Zahra Einalou, ${ }^{a}$ Keivan Maghooli, ${ }^{\text {b,* }}$ Seyaed Kamaledin Setarehdan, ${ }^{c}$ and Ata Akin ${ }^{d}$ \\ aDepartment of Biomedical Engineering, North Tehran Branch, Islamic Azad University, Tehran, Iran \\ ${ }^{b}$ Department of Biomedical Engineering, Science and Research Branch, Islamic Azad University, Tehran, Iran \\ 'University of Tehran, Control and Intelligent Processing Center of Excellence, School of Electrical and Computer Engineering, \\ College of Engineering, Tehran, Iran \\ ${ }^{\mathrm{d}}$ Acibadem University, Department of Medical Engineering, Istanbul, Turkey
}

\begin{abstract}
Functional near-infrared spectroscopy (fNIRS) has been proposed as an affordable, fast, and robust alternative to many neuroimaging modalities yet it still has long way to go to be adapted in the clinic. One request from the clinicians has been the delivery of a simple and straightforward metric (a so-called biomarker) from the vast amount of data a multichannel fNIRS system provides. We propose a simple-straightforward signal processing algorithm derived from $\mathrm{fNIRS}-\mathrm{HbO}_{2}$ data collected during a modified version of the color-word matching Stroop task that consists of three different conditions. The algorithm starts with a wavelet-transform-based preprocessing, then uses partial correlation analysis to compute the functional connectivity matrices at each condition and then computes the global efficiency values. To this end, a continuous wave 16 channels fNIRS device (ARGES Cerebro, Hemosoft Inc., Turkey) was used to measure the changes in $\mathrm{HbO}_{2}$ concentrations from 12 healthy volunteers. We have considered $10 \%$ of strongest connections in each network. A strong Stroop interference effect was found between the incongruent against neutral condition $(p=0.01)$ while a similar significance was observed for the global efficiency values decreased from neutral to congruent to incongruent conditions $[F(2,33)=3.46, p=0.043]$. The findings bring us closer to delivering a biomarker derived from fNIRS data that can be reliably and easily adopted by the clinicians. $\odot 2017$ Society of Photo-Optical Instrumentation Engineers (SPIE) [DOI: 10.1117/1.NPh.4.4.041407]
\end{abstract}

Keywords: weighted connectivity graph; global efficiency; Stroop task; wavelet-based partial correlation.

Paper 17050SSR received Apr. 6, 2017; accepted for publication Jul. 19, 2017; published online Aug. 21, 2017.

\section{Introduction}

Functional near-infrared spectroscopy (fNIRS) has been proposed as a noninvasive, rapid, and affordable alternative of functional neuroimaging modality to fMRI and positron emission tomography (PET). Although this technique was introduced and developed in the last three decades, few scientists recently have allocated their efforts on this issue. For establishing clinical applications, the major parts of their attempts have focused on the amendments of sensitivity and specificity of this method. In Refs. 1-3, a relatively complete discussion can be found on fNIRS advantages and limitations. Despite its many years of introduction to the community, it has only one significant clinical application: transcranial oximetry as a monitor for perfusion of the brain. ${ }^{4}$ This approach has been readily commercialized and several FDA-approved instruments are already placed in the operation room. Acceptance of the fNIRS system to neuropsychology and neuropsychiatry has not been all that welcome. Although fNIRS offers many advantages over traditional neuroimaging techniques, it still suffers from several physical and technical issues that lead to hesitations by clinicians and researchers in their adaptation of this technique. ${ }^{5,6}$ Despite a lower resolution due to diffusive behavior of light propagation in tissue, fNIRS is attractive because of its noninvasiveness, comfort, and cost-effectiveness. ${ }^{6-11}$ Allowing the tolerance to head motion fNIRS has been used in experiments in which

*Address all correspondence to: Keivan Maghooli, E-mail: k_maghooli@ srbiau. ac.ir subjects make large movements, ${ }^{12}$ or in studies for monitoring cortical activation in response to noxious stimuli in infants, ${ }^{13}$ adults, ${ }^{14}$ patients, ${ }^{15}$ and assessment of human response to pain. ${ }^{16}$

Currently, there are two trends followed by the "fNIRSan" (members of the fNIRS community) in their efforts to gain acceptance of their system by the clinicians: (1) increase spatial and temporal resolution to provide a full brain scanner; hence be a competitor and an attractive alternative to fMRI and (2) optimize the number of channels and provide a metric (a biomarker) that correlates well with the neurovascular coupling physiology during cognitive activity. The first trend requires the development of an expansive and bulky system that is neither versatile nor mobile whereas the second trend aims to develop a system that is mobile, flexible, inexpensive yet crude and may lack the necessary accuracy of its competitor. However, the second trend also bestows the highest hope of providing a clinically relevant marker. The researchers try to align themselves with one of these camps in their efforts to push this system into the clinic. We have chosen to be allies with the second camp and propose a metric that can be used effectively in evaluating the cognitive state of the individual with respect to his/her physiological finding derived from fNIRS measurements.

The best way to find a clinical marker is to extract meaningful information from the recorded fNIRS data. To reach this goal, many researchers have adapted innovative signal processing algorithms to extract features that can be readily used as these markers. ${ }^{7,17-23}$ In fact, after the success of functional

$2329-423 X / 2017 / \$ 25.00$ (C) 2017 SPIE 
connectivity studies using fMRI, similar studies using fNIRS have emerged recently. ${ }^{7,24}$ Recent studies have shown obviously that human brain connectome networks can be constructed using fMRI, ${ }^{32}$ diffusion tensor imaging, ${ }^{33}$ electroencephalography (EEG), ${ }^{34}$ and magnetoencephalography (MEG) data, ${ }^{35,36}$ and even further be investigated by graph theoretical approaches. ${ }^{37}$

In this study, we aimed to investigate the efficiency of the graphs formed in the prefrontal cortex (PFC) during a modified version of the color-word matching Stroop task. ${ }^{30,38}$ Connectivity patterns obtained from the partial correlations (PC) of the hemodynamic responses acquired from 16 different regions of the PFC to various stimuli are expected to be different from channel to channel and this is related with the cognitive load. ${ }^{19,39,40} \mathrm{~A}$ continuous wave 16 channels near-infrared spectroscopy device (ARGES Cerebro, Hemosoft Inc., Turkey) was used to measure the changes in $\mathrm{HbO}_{2}$ concentrations from 12 healthy volunteers.

An important aspect of our research is the exploration of the potential of fNIRS to be used as a functional neuroimaging modality ${ }^{19}$ in an expectation to deliver a clinical marker. The second important contribution of our research is the introduction of a simple and straightforward signal-processing algorithm: a wavelet-based partial correlation (WPC) analysis that allowed us to focus on the functional connectivity in a well-defined frequency interval $(0.0035$ to $0.08 \mathrm{~Hz})$ while eliminating the use of high order digital filters and preprocessing techniques that end up warping the data dramatically. Use of wavelet transform-based elimination of nonneural sources of noise, such as cardio respiratory effects, has been quite successful in fNIRS signal-processing applications. ${ }^{30,41-43}$ Moreover, WPC analysis helps to remove the effect of indirect paths; since by applying this method while the WPC between two channels is correlated, an underlying correlation common to all other 14 regions can be regressed out.

Hence the main aim of this study is to explore the feasibility of fNIRS to monitor the neural correlates of the cognitive activity in PFC during a Stroop test by using wavelet analysis and graph theory. The choice of a wavelet-based filtering can in fact be more justified for real-time analysis since wavelets are excellent choices for real-time applications. Usually real-time neurofeedback systems require several seconds of data recording before proving feedback. Hence a collection of initial 10 to $20 \mathrm{~s}$ of data for real-time feedback would be ideal and then every new second the previous $20 \mathrm{~s}$ would be analyzed to provide the feedback. Correlation and GE calculations are very fast algorithms that can be computed in the millisecond range by any DSP chip.

\section{Materials and Methods}

\subsection{Subjects and Protocol}

Data were collected from 12 healthy volunteers ( 7 females and 5 males) from the university community (right-handed, mean age $26.17 \pm 4.30$, range 20 to 31 ) at the Neuro-Optical Imaging Laboratory, Bogazici University Istanbul, Turkey. Control subjects had no history of psychiatric or neurological disorders. Subjects were asked to perform color-word matching Stroop tasks whose trials are the Turkish versions of Zysset et al. ${ }^{38,44}$ Subjects were presented with two words, one written above the other. The top one was written in ink color whereas the bottom one was in white. Subjects were asked to judge whether the word written below correctly denotes the color of the upper word or not. If color and word match, then subjects were to press on the left mouse button with their forefinger, and if not, on the right mouse button with their middle finger.
Color-word Stroop

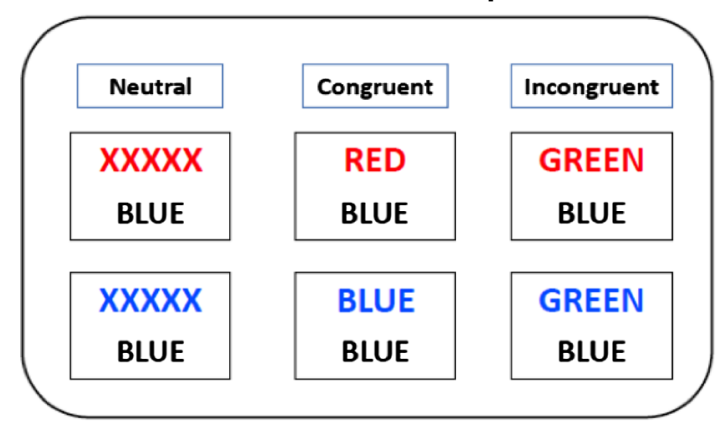

Fig. 1 Stroop task protocol: the subjects responded to each stimulus in a 4-s interval.

Subjects were informed to perform the task as quickly and correctly as possible. The words stayed on the screen until the response was given with a maximum time of $3 \mathrm{~s}$. The screen was blank between the trials. The experiment consisted of neutral $(\mathrm{N})$, congruent $(\mathrm{C})$, and incongruent (IC) trials. In the neutral condition $(\mathrm{N})$, the upper word consisted of four X's (XXXX) in ink color. In the congruent condition (C), ink colors of the upper word and the word itself were the same, whereas in the incongruent condition (IC), they were different. The trials were presented in a semiblocked manner (see Fig. 1 for the details). Each block consisted of six trials. The interstimulus interval within the block was $4.5 \mathrm{~s}$ and the blocks were placed $20 \mathrm{~s}$ apart in time. The trial type within a block was homogeneous (but the arrangements of false and correct trials were altering). There were five blocks of each type. The experiments were performed in a silent, lightly dimmed room. Words were presented via an LCD screen that was $0.5 \mathrm{~m}$ away from the subjects. The task protocol was approved by the Ethics Review Board of Bogazici University. $7,19,45,46$

\subsection{Data Acquisition}

The fNIRS device is capable of transmitting near-infrared light at two wavelengths (730 and $850 \mathrm{~nm}$ ), which are known to be able to penetrate through the scalp and probe the cerebral cortex. Employing 4 LEDs and 10 detectors, the device can sample 16 different channels in the brain simultaneously (see Fig. 2 for the details of the probe ${ }^{47}$ ). LEDs and detectors were placed in a flexible printed circuit board that was specially designed to fit the curvature of the forehead. The source-detector separation is fixed at $2.5 \mathrm{~cm}$, which is optimized for penetration depth $\left(\sim 1.5 \mathrm{~cm}\right.$ from the surface to allow sampling from the cortex $\left.{ }^{48}\right)$ and a wider sampling area of the PFC. ${ }^{7,8,49-53}$ Sampling frequency of the device was $1.7 \mathrm{~Hz}$. Calculation of concentration changes of oxy- $\mathrm{Hb}$ and deoxy-Hb in blood is based on a modified version of the Beer--Lambert law. $7,8,20,54$

\subsection{Signal Processing Algorithm}

The signal processing algorithm proposed is summarized in Fig. 3.

As can be seen, this is a consolidation of techniques proposed in the literature by many others. The wavelet transform has been proposed to remove artifacts and irrelevant physiological signals. ${ }^{30,36,41,42,55-57}$ The choice of the details and approximation coefficients was based on the spectral analysis of fNIRS signals. ${ }^{7,19,20,28,58-60}$ 


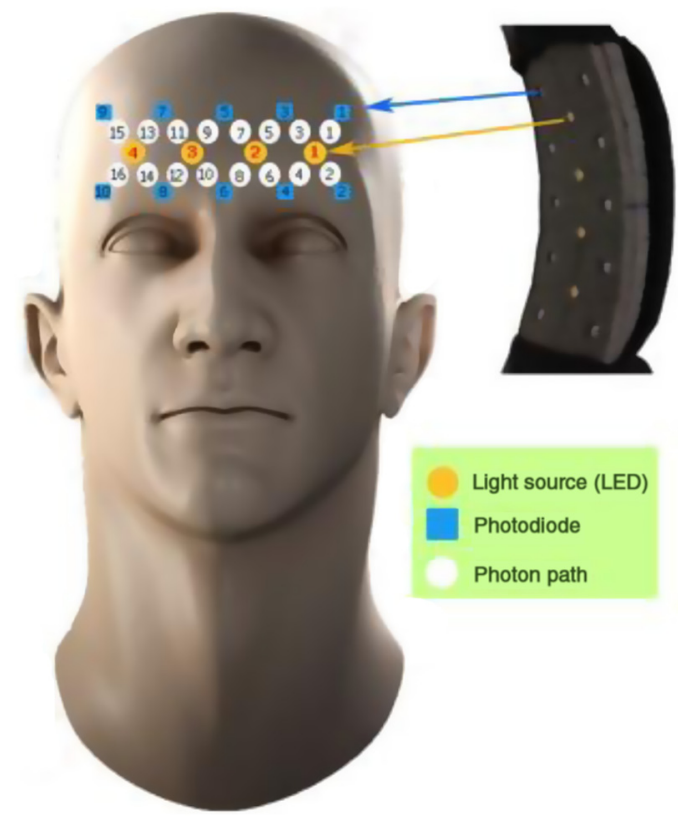

Fig. 2 Details of the fNIRS probe and its approximate placement on the forehead. ${ }^{47}$

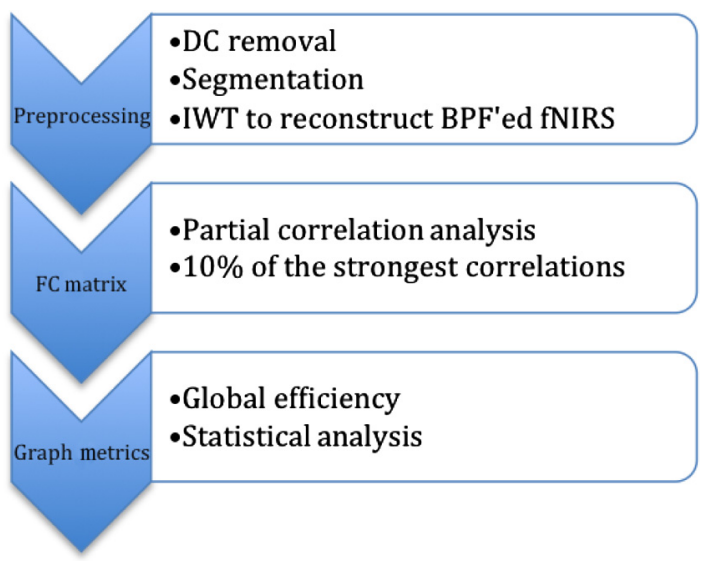

Fig. 3 Signal processing steps used in generation of the global efficiency metric. DC, direct current (baseline signal value); IWT, inverse wavelet transform; BPF, band pass filter; FC, functional connectivity.

\subsubsection{Wavelet transform-based preprocessing}

The discrete wavelet transform (DWT) uses filter banks to perform the wavelet analysis. The DWT decomposes the signal into wavelet coefficients that can represent the signal in various frequency bands. The choice of wavelet function plays an important role in the quality of the analysis of fNIRS signals. Similarity of the mother wavelet to the hemodynamic response function improves the detectability of the estimation accuracy of the signal. ${ }^{42}$ Hence, the decomposition was done by the Daubechies 5 (Db5) as the mother wavelet because of its high similarity to hemodynamic response..$^{42,61}$ The wavelet domain is advantageous to focus in a defined frequency interval to emphasize the functional association between brain regions subtended by cognitive activity. Neuronal activity-related hemodynamic response observed in $\mathrm{fNIRS}-\mathrm{HBO}_{2}$ data are shown to occupy the frequency range of $(0.003$ to $0.08 \mathrm{~Hz}){ }^{22,28,45,46}$ According to the wavelet decomposition tree, the frequency band of the hemodynamic response falls in the approximation at level 3 (CA3: 0.0035 to $0.110 \mathrm{~Hz}$ ) with respect to the sampling frequency of the fNIRS instrument. The coefficients at very low and higher frequency values were nulled and then a new fNIRS- $\mathrm{HBO}_{2}$ signal was reconstructed for each detector.

\subsubsection{Partial correlation}

PC is a useful statistical tool for determining the relationship between two variables after removing the effects of other variables. ${ }^{62,63}$ The goal of PC analysis is to figure out the hidden relation between two channels, while the interactions of other channels on them have been eliminated. In our study, 16 channels were used to investigate the functional connectivity $(D=16)$. Suppose that $x=\left(x_{i}\right)_{i=1, \ldots D}$ are the time signals related to each of the 16 channels of the fNIRS signal. The PC coefficient between the two channels $i$ and $j$ is defined by $\Pi_{i, j}$, which is a calculation of the conditional correlation between channels $i$ and $j$ irrespective of the effect of $D-2$ remaining channels $(R /\{(i, j)\})$.

$$
\Pi_{i, j}=\operatorname{corr}\left[x_{i}, x_{j} \mid x_{R \backslash\{i, j\}}\right] .
$$

It has been shown that the PC matrix can be obtained by calculating the covariance matrix $(\Sigma)$ from $D$ channels. ${ }^{38,39}$ Thus, for $D=16$, the number of PC coefficients will be equal to $D(D-1) / 2=120$, which is obtained from the PC matrix. Matrix $\Pi$ can be easily calculated through the reverse of covariance matrix $\left(\Upsilon=\left[\Upsilon_{i, j}\right]=\Sigma^{-1}\right)$. The reverse covariance matrix of $\mathrm{X}$ is called precision matrix or concentration matrix. ${ }^{47,62-83}$ Thus,

$\Pi_{i, j}=-\frac{\Upsilon_{i j}}{\sqrt{\Upsilon_{i i} \Upsilon_{j j}}}$,

where $i$ and $j$ are the two separate channels and $\Pi_{i i}=1$. The value range of $\mathrm{PC}$ is between +1 and -1 . PC values were computed for each frequency band in three types of stimuli. Since PC analysis helps to remove the effect of indirect paths, by applying this method, the PC between two channels is correlated with the activity at all other 14 regions regressed out. ${ }^{62,63}$ By applying this method, we investigated connectivity graphs based on PCs between the inverse wavelet transformed (reconstructed) data that correspond to the specific frequency band of interest.

\subsubsection{Weighted connectivity graph}

Graph-based network analysis represents the state-of-the-art methodology in brain connectivity. We considered the channels as a set of vertices $V$ and the PC coefficients assigned weights on the set of edges $E$, leading to an undirected complete weighted graph $G=(V ; E) .^{21,56,64}$ We investigated the connectivity graphs of the PCs computed between wavelet coefficients at the third level (corresponding to 0.0035 to $0.110 \mathrm{~Hz}$ frequency range) of each channel. The graphs were computed for each stimulus type.

\subsubsection{Global efficiency and cost of a graph}

Efficiency can be evaluated for a wide range of networks, including weighted graphs. The formal definition is as follows:

$\mathrm{GE}=\frac{1}{N(N-1)} \sum_{i \neq j \in G} \frac{1}{L_{i j}}$, 
where $N$ is the number of nodes in the network, $L_{i j}$ is the shortest path length between nodes $i$ and $j .{ }^{56}$ Maximal possible global efficiency (GE) occurs when all edges are present in the network. We used Latora and Marchiori's ${ }^{65}$ efficiency measure, since it allows us to work with weighted connectivity graphs. In this case, the GE is computed as

$\mathrm{GE}=\frac{1}{N(N-1)} \sum_{i \neq j \in G} \frac{1}{d_{i j}}$,

where $d_{i j}$ is "defined as the smallest sum of the physical distances throughout all the possible paths in the graph from $i$ to $j . "{ }^{\prime 65}$ This has an interpretation, as stronger connection weights intuitively correspond to shorter lengths. Equation (4) generates values of GE in the range of $[0 ; \infty]$. This value can be normalized to $[0 ; 1]$ by dividing it into randomly generated networks with the same number of nodes. This analysis provides an insight to the robustness of the network and its closeness to small network properties. ${ }^{32,55,66}$ We generated 100 degree matched random networks to compute the ratios of global efficiency (GE/GE $\mathrm{Grandom})$ between the real brain functional networks and 100 degree matched random networks to assess small-worldliness of brain functional networks. Typically, GE of a small world network (GESW) approximates to the GE of a random network (GESW/GE $\mathrm{Gandom}_{\text {ra }} \sim 1$ ).

We, then, investigated the topological properties of the brain functional network as a function of GE and $K_{\text {cost }}$. The total number of edges in a graph divided by the maximum possible number edges $N(N-1) / 2$

$K_{\mathrm{cost}}=\frac{1}{N(N-1)} \sum_{i \in G} K_{i}$

is called the cost of the network, which measures how expensive it is to build the network. ${ }^{65}$ The degree of each node, $K_{i}$, $i=1,2, \ldots, 16$, is defined as the number of nodes in the subgraph $G_{i}$. A subgraph $G_{i}$ is defined as the graph including the nodes that are the direct neighbors of the $i$ 'th node. $K_{\text {cost }}$ is the average of the degrees of all the nodes in the graph, which is a measure for the sparsity of a network.

\subsubsection{Behavioral results}

To achieve the behavioral results, we analyzed the reaction times (RTs) from data of 12 subjects. In order to compare each pair of stimuli, we apply a two-tailed paired $t$-test for RTs. The interference effect for the RTs between IC and $\mathrm{N}$ conditions ( $p=0.01)$ and facilitation effect for the RTs of $\mathrm{N}$ against $\mathrm{C}$ conditions are significantly different $(p=0.03)$. However, there is no significant difference between RT of IC and C ( $p=0.8)$.

\section{Results}

\subsection{Global Efficiency Results}

We averaged across all 100 generated random networks to obtain a mean $\overline{\mathrm{GE}}_{\text {random }}$ for each degree $K$ and threshold $T{ }^{67}$ Over a wide range of cost $(0.005<K<0.04)$, results are consistent with previous functional brain network studies. ${ }^{55,68}$ Figure 4(a) shows GE values for N, C, and IC in a real network (average of 12 subjects) and random networks for each $K$ cost.

We investigated the small-world that corresponded to the degree of connectivity threshold $0.01<T<0.99$ (with steps of
0.01). Analysis of variance (ANOVA) was used to test the statistical significance among GE values from different stimulus types (i.e., $\mathrm{GE}_{\mathrm{N}}, \mathrm{GE}_{\mathrm{C}}$, and $\mathrm{GE}_{\mathrm{IC}}$ ). Figure $4(\mathrm{~b})$ shows significance at six different threshold values. The purple triangles indicate that there are significant differences between the GE of three types of stimuli $(p<0.05)$. We investigated the topological properties of the brain functional network as a function of GE and $K$, following the studies by Stam et al. ${ }^{69}$ and Liao et al. ${ }^{70}$

The choice of a threshold value will have a major effect on the topological properties of the resulting networks. This allowed us to compare the topological properties among the three types of stimuli in a manner that is relatively independent of the network size. The threshold is selected to ensure that brain networks have a lower GE compared to random networks with relatively the same degree of connectivity distribution. Ten percent of the strongest connections in each network (highest values of wavelet PC) is considered, which corresponded to the degree of connectivity threshold [see Fig. 4(b)]. GE values were computed for each stimulus condition. Path length is inversely related to the GE of a network for the transfer of information among nodes by multiple parallel paths, and that GE is easier to estimate than path length when studying sparse networks.

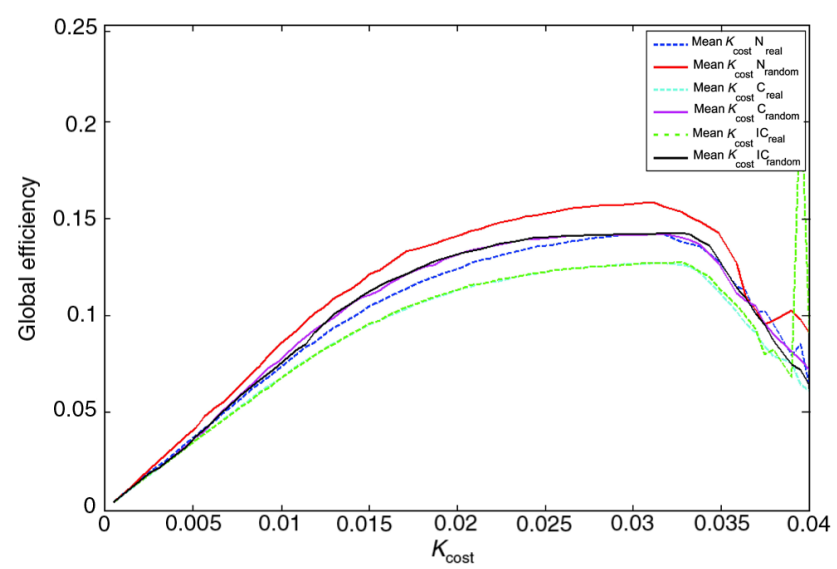

(a)

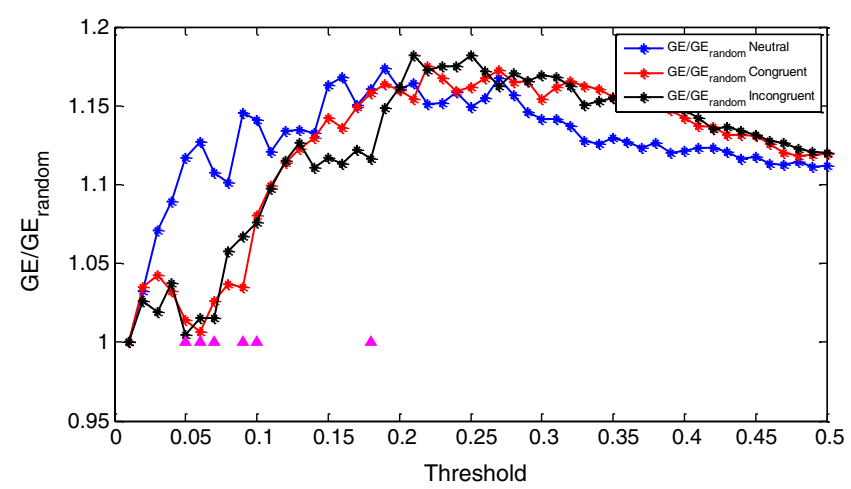

(b)

Fig. 4 (a) The dependence among global efficiency values for three types of stimuli [neutral $(\mathrm{N})$, congruent $(\mathrm{C})$, and incongruent (IC)] and percentage of strongest connections in the network $\left(K_{\text {cost }}\right)$. The solid lines correspond to real network, and the dashed lines correspond to random networks. (b) The ratios of global efficiency (GE/GE $\mathrm{Grndom}_{\text {) }}$ ) between the real brain functional networks and 100 degree matched random networks to assess small-worldliness of brain functional networks. The purple triangles indicate that there are significant differences among the GE of three types of stimuli $(p<0.05)$. 


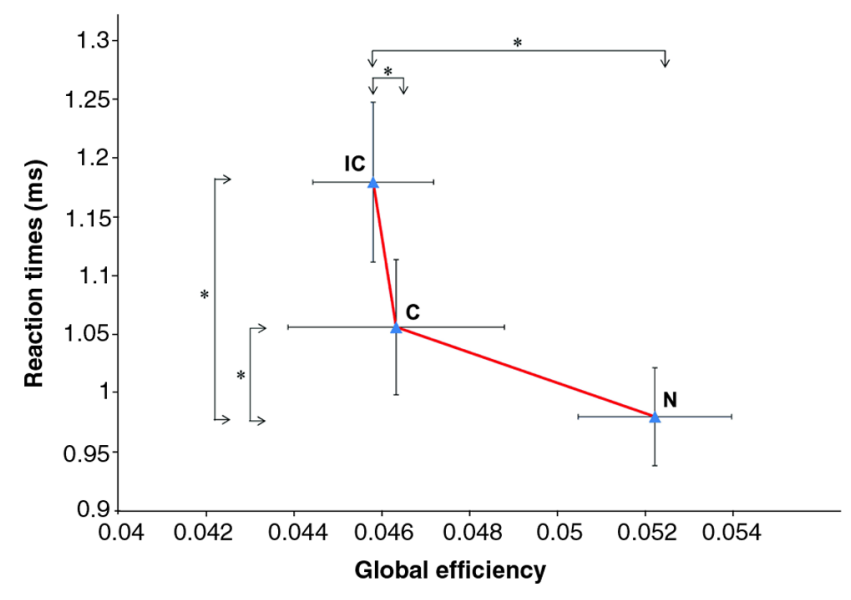

Fig. 5 Mean of GE and RT for three types of conditions. Data are shown as mean standard \pm error (SE). N, neutral task; C, congruent task; IC, incongruent task. Black asterisk indicates $p<0.05$.

GE decreased as the stimulus type became more difficult $[F(2,33)=3.46, \quad p=0.043]$ as seen in Fig. $5\left(\mathrm{GE}_{\mathrm{N}}>\right.$ $\left.\mathrm{GE}_{\mathrm{C}}>\mathrm{GE}_{\mathrm{IC}}\right)$. Although the two-way ANOVA provided a significance, this significance is clearly between the $\mathrm{GE}_{\mathrm{IC}}$ and $\mathrm{GE}_{\mathrm{N}}$ but not between $\mathrm{GE}_{\mathrm{IC}}$ and $\mathrm{GE}_{\mathrm{C}}$ as expected. Normally, the Stroop findings focus on the interference effect (null hypothesis is that the mean value of $\mathrm{GE}_{\mathrm{IC}}$ is the same as the mean value of $\mathrm{GE}_{\mathrm{N}}$ ).

\subsection{GE versus Behavioral Results}

We investigated the relationship between the RTs and GE values in the $\mathrm{N}, \mathrm{C}$, and IC matrices. We computed the change in RTs for different stimulus types and also the change in the GE of the $\mathrm{N}$, $\mathrm{C}$, and IC. Behavioral results and GE analysis according to the stimulus type are shown simultaneously in Fig. 5. The most sought after finding in a color-word matching Stroop task is the contrast of the incongruent condition against the neutral condition. ${ }^{38}$ We show that both in RTs and GE values there is a strong interference effect between the incongruent and neutral conditions.

All in all, we believe that the decreasing GE throughout a task might possess clinical significance.

\section{Discussion}

This study proposes a simple and straightforward algorithmic method to quantify the functional connectivity of the brain using graph theoretical techniques applied to fNIRS- $\mathrm{HBO}_{2}$ signals. We also propose to integrate the well-studied wavelet-based filtering and PC analyses as preprocessing tools before graph efficiency is computed. Graph theory gives us a language for networks. It allows us to define networks exactly and to quantify network properties at many different levels. This quantification is likely to improve further since new graph measures are described regularly.

Mesquita et al. performed an fNIRS study during resting state and then applied correlation analysis to determine the functional connectivity among brain regions. ${ }^{26}$ They used the correlation analysis between two optodes. This approach is prone to incorrect correlations since both data might contain similar trends due to interference from systemic fluctuations. Tachtsidis et al. ${ }^{71}$ have shown how these fluctuations can alter the accuracy of detection and diagnosis if not taken care of. Lindauer et al. have proven that the underlying cerebrovascular dynamics are greatly affected by the metabolism, pharmacological interventions, and even diseases and hence hinder the accurate estimation of the neuronal response. ${ }^{25}$ In recent years, several investigators decided to tackle this problem either by proposing optical probe geometries ${ }^{18,20,72-74}$ or advanced signal processing techniques. ${ }^{19,22,29,42,62,63}$ While a remedy to this interference problem might be adding optodes with short separation, it comes at a cost of increased device complexity and expense. Signal processing tools have shown to reliably improve this desensitization to superficial fluctuations with a common physiological background. Tak and Ye have provided an excellent review on the capabilities of such alternatives. ${ }^{17}$ The nature of this interference is the basic underlying physiology and it can be assumed to be present with a varying degree of contamination in each and every time series of fNIRS data. One alternative to eliminate this contamination and achieve nuisance-free fNIRS data representing the neural activity is the use of PC analysis. ${ }^{24,62}$ The method we used in this study based on PC eliminates this deceiving correlation by considering the similar trends underlying other optode data and eliminating them. This approach also provides a means to bypass any unnecessary preprocessing of the data. Among many denoising algorithms, we decided to use the wavelet transform-based approach since the effectiveness of this technique has been shown in many cases where there might be spike-like or stepwise motion artifacts in the signal. Such artifacts will not be removed by any bandpass infinitive impulse response filtering technique and in fact lead to a more fatal distortion in the signal as the smearing of that spike onto the adjacent time points. Yet another advantage of waveletbased denoising is its use of very short duration filters causing minimal phase distortion possibly minimizing an error in calculation of the correlation coefficient. ICA can be considered as an alternative yet the assumption in ICA-based approaches is that the sources are already independent of each other. The noise might be independent but the systemic physiological background signal is not. Hence, it is nearly impossible to find ICA sources that are band limited yet uncorrelated with the noise. So the natural choice was a wavelet-based approach.

Graph theoretical metrics have been applied to many neuroimaging data especially from fMRI. ${ }^{21,36,37,67}$ Studies by Supekar et al. have shown that functional connectivity computed by the correlation approach provides clinically significant value specifically in Alzheimer's patients. ${ }^{57}$ Studies by Skidmore et al. have shown functional connectivity computed by wavelet correlation analysis in individuals with idiopathic Parkinson's disease. ${ }^{56}$ A graph theoretical metric, GE, was used in discriminating the functional connectivity patterns observed in these patients. We used the same metric to investigate further in healthy people how this metric is influenced among various cognitive loads. In contrast to these fMRI studies, we performed an analysis to prove that fNIRS can reliably be applied to obtain these metrics. Recently, Niu et al. used the graph theoretical network analysis approach to examine the topological organization of the human whole-brain functional network constructed using resting-state fNIRS data. ${ }^{27,31,54}$ Studies by Liu et al. have shown that functional connectivity computed again by the PC approach provides clinically significant values specifically in schizophrenia patients. ${ }^{80}$ Previous fNIRS studies have shown that patients with schizophrenia have impaired activity in the PFC. Taniguchi et al. measured reduced brain activity in PFC of schizophrenia 
patients compared to healthy controls during the Stroop task. ${ }^{81}$ GE has an intuitive interpretation, as higher connection weights intuitively correspond to shorter lengths.

In contrast to studies emphasizing whole-brain network, we found that data from a specific region (i.e., PFC) can be used to generate a global connectivity metric during cognitive tasks. As Eq. (4) dictates, GE is "the average inverse shortest path length" that may significantly contribute to an integration in larger and sparser networks. GE values are inversely related to link weights, as large weights typically represent strong associations, so might GE decrease as the cognitive activity becomes more demanding. GE results are consistent with the hypothesis that information transfer among the regions of PFC will increase with the increasing cognitive load. Path length provides a measure of the network's capacity for serial information transfer among nodes, whereas global efficiency is a measure of the network's capacity for parallel information transfer among nodes via multiple series of edges. Since the evidence is strong that the brain is already massively parallel processing, it seems preferable to adopt comprehensive measures of the efficiency of the brain's functional network topology. ${ }^{55}$ Among various graph theory metrics, we focused on the GE as a marker of the engagement of the PFC with respect to cognitive load since on a global scale, GE quantifies the exchange of information across the whole network where information is concurrently exchanged while local efficiency quantifies a network's resistance to failure on a small scale. That is, the local efficiency of a node characterizes how well information is exchanged by its neighbors when it is removed. Since PFC is usually considered to have been one large network, it would seem only reasonable to treat it as one network and so we focused on a metric that would provide the connectivity of the whole network. ${ }^{82,83}$ Intrinsic functional networks of the human brain have been generated by EEG, fMRI, or MEG modalities and they all demonstrate a converging and highly conserved topological organization over different scales, such as small-world and modular structures..$^{21,33,55,57,65,66,70}$ More importantly, some of these features exhibit specific changes associated with normal development, aging, and various pathological attacks, which indicates the potential value of these approaches in capturing and monitoring the brain organization under different mental states. ${ }^{1,3,4,25-31,60,75}$ Our findings on the decrease of GE as the cognitive demand increases might sound counterintuitive since a major hypothesis of network theory is that an adaptive network should reorganize itself to minimize its cost and increase efficiency under increasing load. Considering the increase in RT to represent an increase in cognitive load, we see a consecutive decrease in the GE values. There might be several explanations for this finding: (1) a flaw with the signal processing methodology and (2) a lack in observing only a piece of a larger network. The PC algorithm we employed uses the remaining 14 channels data as regressors for computing the correlation between the two channels. It is quite possible that this approach might be leading to an over regression (removing too much of the dependence) from the channels leading to a smaller correlation value. Hence the functional connectivity matrix calculated after this operation might leave only the very close channels as strongly correlated. This will eventually lead to a lower GE value. Second, PFC is a part of a larger brain network, albeit its fundamental role in decision-making. fNIRS has access only to this region and it might be quite possible that as the cognitive demand increases many other parts of the brain might be employed that are not visible to fNIRS sampling. Hence, we might be observing a piece of a network and the cognitive demand could be distributed over other parts of the brain. That could also explain a decrease in GE as the cognitive load increases.

\subsection{Limitation of the Study}

We reiterate the rationale behind our choice of this sourcedetector separation. This has been a long debate and many authors have favored the use of source-detector separations as close as $2.5 \mathrm{~cm}$. In fact one of the pioneers of fNIRS, the late Dr. Britton Chance himself has used rectangular probe geometry with an SD separation of $2.5 \mathrm{~cm}$ in most of his studies. We would like to bring to your attention to a paragraph from his one of the most cited articles. ${ }^{11}$ The major intracerebral contribution probably comes from the gray matter. ${ }^{65}$ This has been confirmed in two studies performing PET and NIRS simultaneously that have shown the best correlation between NIRS and PET parameters in the outer $1 \mathrm{~cm}$ of the brain tissue. ${ }^{11,79}$ Interestingly, it seems that even at interoptode distances as short as 2 to $2.5 \mathrm{~cm}$ gray matter is part of the sample volume. ${ }^{76}$ This is consistent with work assessing changes in local brain activity successfully with interoptode distances of $2.5 \mathrm{~cm} .{ }^{77}$ Other authors have reported measurements at even smaller interoptode distances. ${ }^{78}$

Our group has also shown through Monte Carlo simulations run on a realistic head model that we can actually probe the gray matter. ${ }^{48}$ We agree that the probed gray matter area will increase when the source-detector separation is enlarged to $3 \mathrm{~cm}$, albeit at the cost of reduction in SNR. Even at an SD separation of 2.5 we are losing $\sim 1 / 10^{6}$ of the photons. Hence, this choice becomes an optimization issue. Even though only about $2 \%$ to $3 \%$ of the signal we collect comes from the gray matter, the dynamical changes observed and extracted with proper signal processing techniques correlate significantly with the task. Hence as engineers we are faced with the dilemma ensuring a deeper penetration depth via a larger SD separation at the expense of complexity and cost of equipment and bulkiness of the probe, or choosing an optimized distance at the expense of lesser probing of the gray matter but a higher SNR and far less inexpensive and complex instrumentation.

\section{Conclusion}

In this study, a modified version of the color-word matching Stroop task was employed during fNIRS data collection. The aim was to elucidate the adaptation of brain connectivity patterns in the PFC during the task. The data were preprocessed by WPC and local efficiency values were assessed among the 16 different regions. The findings show promise when interference between incongruent and neutral conditions is considered. The simple yet straightforward signal processing approach proposed may lead to new findings in the assessment of connectivity changes for diagnostic and prognostic purposes. The choice of this specific signal processing algorithm was motivated from the literature findings where a convergence was observed to a wavelet-based elimination of irrelevant physiological background activity and some instrumentation noise. The choice of PC to compute the functional connectivity matrix was motivated by the need to eliminate a common background systemic physiological activity that can be observed in each recording. The study is limited in its choice of the graph theoretical metrics to only global efficiency. Although many metrics could have been employed, we believe that global efficiency actually is the 
major metric since it is derived from other metrics of graph theory.

\section{Disclosures}

No conflicts of interest, financial or otherwise, are declared by the authors.

\section{References}

1. F. Scholkmann et al., "A review on continuous wave functional nearinfrared spectroscopy and imaging instrumentation and methodology," Neuroimage 85, 6-27 (2014).

2. M. Wolf, M. Ferrari, and V. Quaresima, "Progress of near-infrared spectroscopy and topography for brain and muscle clinical applications," J. Biomed. Opt. 12(6), 062104 (2007).

3. F. Irani et al., "Functional near infrared spectroscopy (fNIRS): an emerging neuroimaging technology with important applications for the study of brain disorders," Clin. Neuropsychol. 21(1), 9-37 (2007).

4. M. T. Douds et al., "A systematic review of cerebral oxygenation-monitoring devices in cardiac surgery," Perfusion 29(6), 545-552 (2014).

5. H. Obrig and A. Villringer, "Beyond the visible-imaging the human brain with light," J. Cereb. Blood Flow Metab. 23(1), 1-18 (2003).

6. H. Obrig et al., "Near-infrared spectroscopy: does it function in functional activation studies of the adult brain?" Int. J. Psychophysiol. 35(2), 125-142 (2000).

7. S. Aydöre et al., "On temporal connectivity of PFC via Gauss-Markov modeling of fNIRS signals," IEEE Trans. Biomed. Eng. 57(3), 761-768 (2010).

8. B. Chance et al., "A novel method for fast imaging of brain function, non-invasively, with light," Opt. Express 2(10), 411-423 (1998).

9. I. Palada et al., "Cerebral and peripheral hemodynamics and oxygenation during maximal dry breath-holds," Respir. Physiol. Neurobiol. 157(2), 374-381 (2007).

10. D. T. Ubbink and B. Koopman, "Near-infrared spectroscopy in the routine diagnostic work-up of patients with leg ischaemia," Eur. J Vasc. Endovasc. Surg. 31(4), 394-400 (2006).

11. A. Villringer and B. Chance, "Non-invasive optical spectroscopy and imaging of human brain function," Trends Neurosci. 20(10), 435442 (1997).

12. X. Cui, S. Bray, and A. L. Reiss, "Functional near infrared spectroscopy (NIRS) signal improvement based on negative correlation between oxygenated and deoxygenated hemoglobin dynamics," Neuroimage 49(4), 3039-3046 (2010).

13. M. Bartocci et al., "Pain activates cortical areas in the preterm newborn brain," Pain 122(1), 109-117 (2006).

14. L. Becerra et al., "Diffuse optical tomography activation in the somatosensory cortex: specific activation by painful vs. non-painful thermal stimuli," PLoS One 4(11), e8016 (2009).

15. C. Gélinas et al., "Toward a new approach for the detection of pain in adult patients undergoing cardiac surgery: near-infrared spectroscopya pilot study," Heart Lung: J. Acute Crit. Care 39(6), 485-493 (2010).

16. Z. Barati et al., "Hemodynamic response to repeated noxious cold pressor tests measured by functional near infrared spectroscopy on forehead," Ann. Biomed. Eng. 41(2), 223-237 (2013).

17. S. Tak and J. C. Ye, "Statistical analysis of fNIRS data: a comprehensive review," Neuroimage 85, 72-91 (2014).

18. Q. Zhang, E. N. Brown, and G. E. Strangman, "Adaptive filtering for global interference cancellation and real-time recovery of evoked brain activity: a Monte Carlo simulation study," J. Biomed. Opt. 12(4), 044014 (2007).

19. K. Ciftçi et al., "Multilevel statistical inference from functional nearinfrared spectroscopy data during Stroop interference," IEEE Trans. Biomed. Eng. 55(9), 2212-2220 (2008).

20. C. B. Akgül, A. Akin, and B. Sankur, "Extraction of cognitive activityrelated waveforms from functional near-infrared spectroscopy signals," Med. Biol. Eng. Comput. 44(11), 945-958 (2006).

21. Y. He and A. Evans, "Graph theoretical modeling of brain connectivity," Curr. Opin. Neurol. 23(4), 341-350 (2010).

22. T. J. Huppert et al., "HomER: a review of time-series analysis methods for near-infrared spectroscopy of the brain," Appl. Opt. 48(10), D280D298 (2009).
23. M. L. Schroeter et al., "Towards a standard analysis for functional nearinfrared imaging," NeuroImage 21(1), 283-290 (2004).

24. Z. Einalou et al., "Functional near infrared spectroscopy to investigation of functional connectivity in schizophrenia using partial correlation," Univers. J. Biomed. Eng. 2(1), 5-8 (2014).

25. U. Lindauer et al., "Pathophysiological interference with neurovascular coupling-when imaging based on hemoglobin might go blind," Front. Neuroenerg. 2, 25 (2010).

26. R. C. Mesquita, M. A. Franceschini, and D. A. Boas, "Resting state functional connectivity of the whole head with near-infrared spectroscopy," Biomed. Opt. Express 1(1), 324-336 (2010).

27. H. Niu et al., "Test-retest reliability of graph metrics in functional brain networks: a resting-state fNIRS study," PLoS One 8(9), e72425 (2013).

28. S. Sasai et al., "Frequency-specific functional connectivity in the brain during resting state revealed by NIRS," NeuroImage 56(1), 252-257 (2011).

29. H. Zhang et al., "Functional connectivity as revealed by independent component analysis of resting-state fNIRS measurements," NeuroImage 51(3), 1150-1161 (2010).

30. L. Zhang et al., "Studying hemispheric lateralization during a Stroop task through near-infrared spectroscopy-based connectivity," J. Biomed. Opt. 19(5), 057012 (2014).

31. H. Niu and Y. He, "Resting-state functional brain connectivity: lessons from functional near-infrared spectroscopy," Neuroscientist 20(2), 173188 (2014).

32. J. Wang et al., "Parcellation-dependent small-world brain functional networks: a resting-state fMRI study," Hum. Brain Mapp. 30(5), 1511-1523 (2009).

33. M. J. Vaessen et al., "The effect and reproducibility of different clinical DTI gradient sets on small world brain connectivity measures," Neuroimage 51(3), 1106-1116 (2010).

34. S. G. Horovitz et al., "Low frequency BOLD fluctuations during resting wakefulness and light sleep: a simultaneous EEG-fMRI study," Hum. Brain Mapp. 29(6), 671-682 (2008).

35. J. F. Alonso et al., "MEG connectivity analysis in patients with Alzheimer's disease using cross mutual information and spectral coherence," Ann. Biomed. Eng. 39(1), 524-536 (2011).

36. J. F. Alonso et al., "MEG connectivity analysis in patients with Alzheimer's disease using cross mutual information and spectral coherence," Ann. Biomed. Eng. 39(1), 524-536 (2011).

37. J.-H. Wang et al., "Graph theoretical analysis of functional brain networks: test-retest evaluation on short-and long-term resting-state functional MRI data," PLoS One 6(7), e21976 (2011).

38. S. Zysset et al., "Color-word matching Stroop task: separating interference and response conflict," Neuroimage 13(1), 29-36 (2001).

39. A.-C. Ehlis et al., "Multi-channel near-infrared spectroscopy detects specific inferior-frontal activation during incongruent Stroop trials," Biol. Psychol. 69(3), 315-331 (2005).

40. M. L. Schroeter et al., "Near-infrared spectroscopy can detect brain activity during a color-word matching Stroop task in an event-related design," Hum. Brain Mapp. 17(1), 61-71 (2002).

41. U. E. Emir et al. "Wavelet denoising vs. ICA denoising for functional optical imaging." in Conf. Proc. First Int. IEEE EMBS Conf. on Neural Engineering, IEEE (2003).

42. B. Molavi and G. A. Dumont, "Wavelet-based motion artifact removal for functional near-infrared spectroscopy," Physiol. Meas. 33(2), 259270 (2012).

43. M. Dadgostar, S. K. Setarehdan, and A. Akin, "Detection of motion artifacts in fNIRS via the continuous wavelet transform," in 20th Iranian Conf. on Biomedical Engineering (ICBME '13), IEEE (2013).

44. M. L. Schroeter et al., "Age dependency of the hemodynamic response as measured by functional near-infrared spectroscopy," Neuroimage 19(3), 555-564 (2003).

45. Z. Einalou et al., "Functional near infrared spectroscopy for functional connectivity during Stroop test via mutual information," Adv. Biores. 6(1), 62-67 (2015).

46. Z. Einalou et al., "Effective channels in classification and functional connectivity pattern of prefrontal cortex by functional near infrared spectroscopy signals," Optik-Int. J. Light Electron Opt. 127(6), 3271-3275 (2016).

47. M. Dadgostar et al., "Functional connectivity of the PFC via partial correlation," Optik-Int. J. Light Electron Opt. 127(11), 4748-4754 (2016). 
48. S. B. Erdoğan, A. Y. Meryem, and A. Akın, "Analysis of task-evoked systemic interference in fNIRS measurements: insights from fMRI," NeuroImage 87, 490-504 (2014).

49. H. Ayaz et al., "Using MazeSuite and functional near infrared spectroscopy to study learning in spatial navigation," J. Visualized Exp. 56, 3443 (2011).

50. M. Izzetoglu et al., "Functional near-infrared neuroimaging," IEEE Trans. Neural Syst. Rehabil. Eng. 13(2), 153-159 (2005).

51. S. M. Platek et al., "Functional near infrared spectroscopy reveals differences in self-other processing as a function of schizotypal personality traits," Schizophr. Res. 73(1), 125-127 (2005).

52. A. C. Ruocco et al., "Medial prefrontal cortex hyperactivation during social exclusion in borderline personality disorder," Psychiatry Res.: Neuroimaging 181(3), 233-236 (2010).

53. M. Firbank, O. Eiji, and D. T. Delpy, "A theoretical study of the signal contribution of regions of the adult head to near-infrared spectroscopy studies of visual evoked responses," NeuroImage 8(1), 69-78 (1998).

54. H. Niu et al., "Revealing topological organization of human brain functional networks with resting-state functional near infrared spectroscopy," PLoS One 7(9), e45771 (2012).

55. S. Achard and E. Bullmore, "Efficiency and cost of economical brain functional networks," PLoS Comput. Biol. 3(2), e17 (2007).

56. F. Skidmore et al., "Connectivity brain networks based on wavelet correlation analysis in Parkinson fMRI data," Neurosci. Lett. 499(1), 47-51 (2011).

57. K. Supekar et al., "Network analysis of intrinsic functional brain connectivity in Alzheimer's disease," PLoS Comput. Biol. 4(6), e1000100 (2008).

58. H. Obrig et al., "Spontaneous low frequency oscillations of cerebral hemodynamics and metabolism in human adults," Neurolmage 12(6), 623-639 (2000).

59. C. B. Akgül, B. Sankur, and A. Akin, "Spectral analysis of event-related hemodynamic responses in functional near infrared spectroscopy," J. Comput. Neurosci. 18(1), 67-83 (2005).

60. C.-M. Lu et al., "Use of fNIRS to assess resting state functional connectivity," J. Neurosci. Methods 186(2), 242-249 (2010).

61. M. Dadgostar et al., "Comparison of denoising algorithms in fNIRS," in Proc. of the NIRS 2012 Meeting (2012).

62. G. Marrelec et al., "Partial correlation for functional brain interactivity investigation in functional MRI," NeuroImage 32(1), 228-237 (2006).

63. J. Hlinka et al., "Functional connectivity in resting-state fMRI: is linear correlation sufficient?" NeuroImage 54(3), 2218-2225 (2011).

64. E. Bullmore and O. Sporns, "Complex brain networks: graph theoretical analysis of structural and functional systems," Nat. Rev. Neurosci. 10(3), 186-198 (2009).

65. V. Latora and M. Marchiori, "Efficient behavior of small-world networks," Phys. Rev. Lett. 87(19), 198701 (2001).

66. D. J. Watts and S. H. Strogatz, "Collective dynamics of 'small-world' networks," Nature 393(6684), 440-442 (1998).

67. N. Langer, P. Andreas, and L. Jäncke, "The problem of thresholding in small-world network analysis," PLoS One 8(1), e53199 (2013).

68. M.-E. Lynall et al., "Functional connectivity and brain networks in schizophrenia," J. Neurosci. 30(28), 9477-9487 (2010).

69. C. J. Stam et al., "Graph theoretical analysis of magnetoencephalographic functional connectivity in Alzheimer's disease," Brain 132(1), 213-224 (2009).

70. W. Liao et al., "Altered functional connectivity and small-world in mesial temporal lobe epilepsy," PLoS One 5(1), e8525 (2010).

71. I. Tachtsidis et al. "False positives in functional near infrared topography." in Oxygen Transport to Tissue XXX, pp. 307-314, Springer (2009).

72. L. Gagnon et al., "Further improvement in reducing superficial contamination in NIRS using double short separation measurements," NeuroImage 85, 127-135 (2014).
73. L. Gagnon et al., "Short separation channel location impacts the performance of short channel regression in NIRS," NeuroImage 59(3), 25182528 (2012).

74. E. Kirilina et al., "The physiological origin of task-evoked systemic artefacts in functional near infrared spectroscopy," Neurolmage 61(1), 70-81 (2012).

75. A. J. Fallgatter et al., "Nah-Infrarot-spektroskopie in der Psychiatrie," Nervenarzt 75(9), 911-916 (2004).

76. E. Okada et al., "Theoretical and experimental investigation of nearinfrared light propagation in a model of the adult head," Appl. Opt. 36(1), 21-31 (1997).

77. T. Kato et al., "Human visual cortical function during photic stimulation monitoring by means of near-infrared spectroscopy," J. Cereb. Blood Flow Metab. 13(3), 516-520 (1993).

78. Y. O. K. O. Hoshi and M. A. M. O. R. U. Tamura, "Dynamic multichannel near-infrared optical imaging of human brain activity," J. Appl. Physiol. 75(4), 1842-1846 (1993).

79. C. Hock et al., "Decrease in parietal cerebral hemoglobin oxygenation during performance of a verbal fluency task in patients with Alzheimer's disease monitored by means of near-infrared spectroscopy (NIRS)correlation with simultaneous rCBF-PET measurements," Brain Res. 755(2), 293-303 (1997).

80. Y. Liu et al., "Disrupted small-world networks in schizophrenia," Brain 131(4), 945-961 (2008).

81. K. Taniguchi et al., "Multi-channel near-infrared spectroscopy reveals reduced prefrontal activation in schizophrenia patients during performance of the kana Stroop task," J. Med. Invest. 59(1, 2), 45-52 (2012).

82. L. Li et al., "Automated voxel classification used with atlas-guided diffuse optical tomography for assessment of functional brain networks in young and older adults," Neurophoton 3(4), 045002 (2016).

83. T. Fekete et al., "Small-world network properties in prefrontal cortex correlate with predictors of psychopathology risk in young children: a NIRS study," NeuroImage 85, 345-353 (2014).

Zahra Einalou received her $\mathrm{PhD}$ in biomedical engineering from the Science and Research Branch, Islamic Azad University, Tehran, Iran, in 2016. Currently, she works at the Department of Biomedical Engineering at North Tehran Branch, Islamic Azad University as the assistant professor. Her research interests are in the fields of functional neuroimaging and signal processing.

Keivan Maghooli has been the assistant professor at the Department of Biomedical Engineering, Science and Research Branch, Islamic Azad University, Tehran, Iran. $\mathrm{He}$ is a PhD scholar at the mentioned department. He holds his Ms(CS) degree in biomedical engineering from Tarbiat Modares University Tehran, Iran. He has more than 10 years of experience including teaching graduate classes.

Seyed Kamaledin Setarehdan received his $\mathrm{PhD}$ in medical data analysis from the University of Strathclyde in Glasgow, United Kingdom. He was a postdoctoral research fellow in the University of Strathclyde in Glasgow, United Kingdom. In 2001, he joined the School of Electrical and Computer Engineering, College of Engineering, University of Tehran, Iran, where he is currently a professor of biomedical engineering. His main research interests are medical ultrasound and medical applications of the near-infrared spectroscopy.

Ata Akin received his $\mathrm{PhD}$ in biomedical engineering from Drexel University in 1998, his MS degree in biomedical engineering, and his BS degree in electronics and telecommunication engineering both from Istanbul Technical University in 1995 and 1993, respectively. Currently, he works at the Department of Medical Engineering at Acıbadem University and serves as the dean of Faculty of Engineering. His research interests are in the fields of functional neuroimaging and systems biology. 